\title{
Superconductivity and phonon self-energy effects in $\mathrm{Fe}_{1+y} \mathrm{Te}_{0.6} \mathrm{Se}_{0.4}$
}

\author{
S.-F. Wu, ${ }^{1,}{ }^{*}$ A. Almoalem, ${ }^{2}$ I. Feldman, ${ }^{2}$ A. Lee, ${ }^{1}$ A. Kanigel, ${ }^{2}$ and G. Blumberg ${ }^{1,3, \dagger}$ \\ ${ }^{1}$ Department of Physics and Astronomy, Rutgers University, Piscataway, New Jersey 08854, USA \\ ${ }^{2}$ Physics Department, Technion-Israel Institute of Technology, Haifa 32000, Israel \\ ${ }^{3}$ National Institute of Chemical Physics and Biophysics, 12618 Tallinn, Estonia
}

(Received 17 November 2019; revised manuscript received 13 February 2020; accepted 10 March 2020; published 27 March 2020)

\begin{abstract}
We study a $\mathrm{Fe}_{1+y} \mathrm{Te}_{0.6} \mathrm{Se}_{0.4}$ multiband superconductor with $T_{c}=14 \mathrm{~K}$ by polarization-resolved Raman spectroscopy. Deep in the superconducting state, we detect pair-breaking excitation at $2 \Delta=5.6 \mathrm{meV}$ in the $X Y\left(B_{2 g}\right)$ scattering geometry, consistent with the fundamental gap energy $\Delta=3 \mathrm{meV}$ revealed by ARPES on the holelike Fermi pocket with $d_{x z} / d_{y z}$ character. We analyze the superconductivity-induced phonon self-energy effects for the $B_{1 g}(\mathrm{Fe})$ phonon mode and derive the electron-phonon coupling constant $\lambda^{\Gamma} \approx 0.026$, which is insufficient to explain superconductivity with $T_{c}=14 \mathrm{~K}$.
\end{abstract}

DOI: 10.1103/PhysRevResearch.2.013373

\section{INTRODUCTION}

Since the discovery of the multiband iron-based superconductors (FeSCs) in 2008 [1], a unified understanding of the pairing mechanism in FeSCs remains a focus of attention [2-8]. One step towards such understanding is to measure the superconducting (SC) gaps on different pockets of the Fermi surface (FS) [5,9].

The chalcogenide family of $\mathrm{Fe}_{1+y} \mathrm{Te}_{1-x} \mathrm{Se}_{x}$ has a simple stoichiometry and crystal structure which can be viewed as stacks of $\mathrm{FeTe}_{1-x} \mathrm{Se}_{x}$ layers [Fig. 1(a)]. Superconductivity in this system was first found at $9 \mathrm{~K}$ in the nonmagnetic FeSe. With about $60 \%$ isovalent substitution of $\mathrm{Se}$ for $\mathrm{Te}, T_{c}$ in $\mathrm{Fe}_{1+y} \mathrm{Te}_{0.6} \mathrm{Se}_{0.4}$ increases to $14 \mathrm{~K}$ [10]. Thus, the nonmagnetic and tetragonal $\mathrm{Fe}_{1+y} \mathrm{Te}_{0.6} \mathrm{Se}_{0.4}$ is an ideal system to study the SC order parameter, without the effect of coexisting or interacting with other electronic orders.

The polarization-resolved Raman spectroscopy has been used to study bulk properties of the FeSCs: for measurements of the pair-breaking excitations in different symmetry channels of the Raman response which provide direct information on the structure of SC gaps at distinct FS pockets [11-17] and for estimation of the electron-phonon coupling [18-20]. Previous Raman studies on $\mathrm{Fe}_{1+y} \mathrm{Te}_{1-x} \mathrm{Se}_{x}$ were focused on the lattice dynamics [21-25], while the superconducting features for $\mathrm{Fe}_{1+y} \mathrm{Te}_{0.6} \mathrm{Se}_{0.4}$ have not been reliably established [26].

In this article, we use polarization-resolved Raman spectroscopy to study the pair breaking excitations in

\footnotetext{
*sw666@physics.rutgers.edu

${ }^{\dagger}$ girsh@physics.rutgers.edu
}

Published by the American Physical Society under the terms of the Creative Commons Attribution 4.0 International license. Further distribution of this work must maintain attribution to the author(s) and the published article's title, journal citation, and DOI.
$\mathrm{Fe}_{1+y} \mathrm{Te}_{0.6} \mathrm{Se}_{0.4}$. In the SC state, we identify the coherence peak at $45 \mathrm{~cm}^{-1}(2 \Delta=5.6 \mathrm{meV})$ in the $X Y\left(B_{2 g}\right.$ in $\left.D_{4 h}\right)$ scattering geometry with cross-polarized light along $\mathrm{Fe}-\mathrm{Te} / \mathrm{Se}$ directions. The peak energy is consistent with determined by ARPES fundamental gap energy $\Delta=3 \mathrm{meV}$ on the holelike FS pocket around the $\Gamma$ point derived from iron $d_{x z} / d_{y z}$ orbitals [27]. We investigate the superconductivity-induced phonon self-energy effects for the $B_{1 g}(\mathrm{Fe})$ symmetry phonon mode and estimate the electron-phonon coupling constant $\lambda^{\Gamma} \approx 0.026$, which is very weak to explain $T_{c}=14 \mathrm{~K}$ in $\mathrm{Fe}_{1+y} \mathrm{Te}_{0.6} \mathrm{Se}_{0.4}$.

\section{EXPERIMENTAL}

The $\mathrm{Fe}_{1+y} \mathrm{Te}_{0.6} \mathrm{Se}_{0.4}$ single crystals were grown using the modified Bridgman method [27]. The composition was determined on samples from the same growth batch by energydispersive x-ray (EDX) analysis. The nominal composition of excess $\mathrm{Fe}(y)$ in $\mathrm{Fe}_{1+y} \mathrm{Te}_{0.6} \mathrm{Se}_{0.4}$ was about $2 \%$, while the real value of $y$ is close to zero [27]. The magnetic susceptibility confirming a sharp SC transition at $T_{c}=14 \mathrm{~K}$ is shown in Fig. 1(d). The samples from the same batch were investigated by ARPES in Ref. [27].

The $\mathrm{Fe}_{1+y} \mathrm{Te}_{0.6} \mathrm{Se}_{0.4}$ single crystals used for Raman measurements were cleaved in a nitrogen gas atmosphere in a glove bag, then immediately loaded into a connected continuous helium flow optical cryostat and quickly cooled to $250 \mathrm{~K}$ within 5 minutes to avoid surface contamination. All the Raman scattering measurements were performed using the $\mathrm{Kr}^{+}$laser line at $647.1 \mathrm{~nm}(1.92 \mathrm{eV})$ in a quasi-backscattering geometry along the crystallographic $c$ axis with an instrumental resolution about $1.5 \mathrm{~cm}^{-1}$. The excitation laser beam was focused into $50 \times 100 \mu \mathrm{m}^{2}$ spot on the $a b$ surface, with the incident power around 10 and $2 \mathrm{~mW}$ for normal state and superconducting state measurements, respectively. The scattered light was collected and analyzed by a triple-stage aberration-corrected Raman spectrometer and recorded using 
(a)

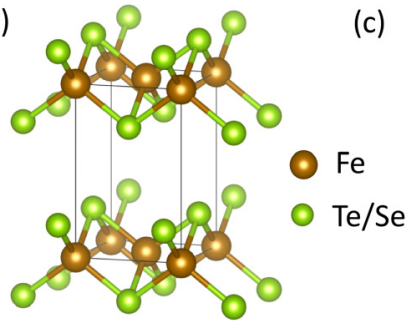

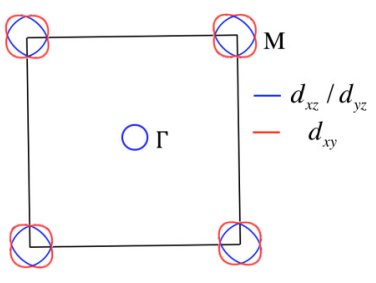

(d)

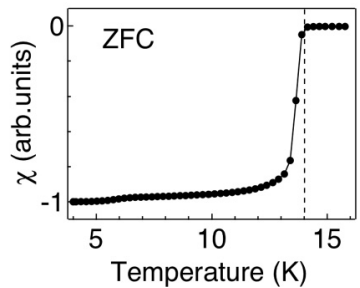

FIG. 1. (a) Crystal structure of $\mathrm{Fe}_{1+y} \mathrm{Te}_{0.6} \mathrm{Se}_{0.4}$. (b) Definition of $X, Y, X^{\prime}$, and $Y^{\prime}$ directions in a $2-\mathrm{Fe}$ unit cell. (c) Schematic representation of Fermi surfaces of $\mathrm{Fe}_{1+y} \mathrm{Te}_{0.6} \mathrm{Se}_{0.4}$ in the 2-Fe Brillouin zone (BZ) $[28,29]$. (d) "Zero-field-cool" magnetic susceptibility measured with magnetic field $\mathrm{H}=4 \mathrm{Oe}$ along the $c$-axis direction.

a liquid nitrogen-cooled charge-coupled detector. The spectra were corrected for the spectral response of the spectrometer and the detector. The derived mode's linewidths were corrected for the instrumental resolution. The temperature shown in this paper has been corrected for laser heating. An empirical heating coefficient $1 \mathrm{~K} / \mathrm{mW}$ was applied according to FeSe [13,30,31].

In this article, we define the $X$ and $Y$ directions along the two-Fe unit cell basis vectors (at $45^{\circ}$ from the $\mathrm{Fe}-\mathrm{Fe}$ directions) in the tetragonal phase, whereas $X^{\prime}$ and $Y^{\prime}$ are along the Fe-Fe directions [Fig. 1(b)]. Raman spectra were recorded for $\left(\hat{e}_{i} \hat{e}_{s}\right)=(X X),(X Y),\left(X^{\prime} X^{\prime}\right)$, and $\left(X^{\prime} Y^{\prime}\right)$ polarization geometries in the $a b$ plane, where $\hat{e}_{i}$ and $\hat{e}_{s}$ represent the incident and scattered light polarization, respectively.

For crystals with point group symmetry $D_{4 h}$, the $X X, X^{\prime} Y^{\prime}$, and $X Y$ geometries probe $A_{1 g}+B_{1 g}, A_{2 g}+B_{1 g}$, and $A_{2 g}+B_{2 g}$ channels, respectively [32]. For the analyses below we assume that the $A_{2 g}$ response is negligible [11]. We estimate a luminescence background $I_{b g}(\omega, T)$ from the spectral intensity in $X^{\prime} Y^{\prime}$-scattering geometry [16]. The Raman response in $(\mu \nu)$ scattering geometry is obtained as $\chi_{\mu \nu}^{\prime \prime}(\omega, T)=\left[I_{\mu \nu}(\omega, T)-\right.$ $\left.I_{b g}(\omega, T)\right] /[1+n(\omega, T)]$, where $n(\omega, T)$ is the Bose-Einstein factor.

\section{A. Phonons}

The Raman active phonon modes for the $\mathrm{Fe}_{1+y} \mathrm{Te}_{0.6} \mathrm{Se}_{0.4}$ structure are $\Gamma_{\text {Raman }}=A_{1 g}+B_{1 g}+2 E_{g}$. The $A_{1 g}$ and $B_{1 g}$ modes correspond to $\mathrm{Te} / \mathrm{Se}$ and $\mathrm{Fe}$ atomic displacements along the $c$-axis direction, respectively.

In Fig. 2 we show Raman spectra for four in-plane scattering geometries at $25 \mathrm{~K}$. The modes at around 162 and $207 \mathrm{~cm}^{-1}$ are assigned to $A_{1 g}(\mathrm{Te} / \mathrm{Se})$ and $B_{1 g}(\mathrm{Fe})$ phonons, respectively $[23,24]$. The comparison of the frequencies and linewidths for these phonons to the previous studies [23,24] are summarized in Table I [33].

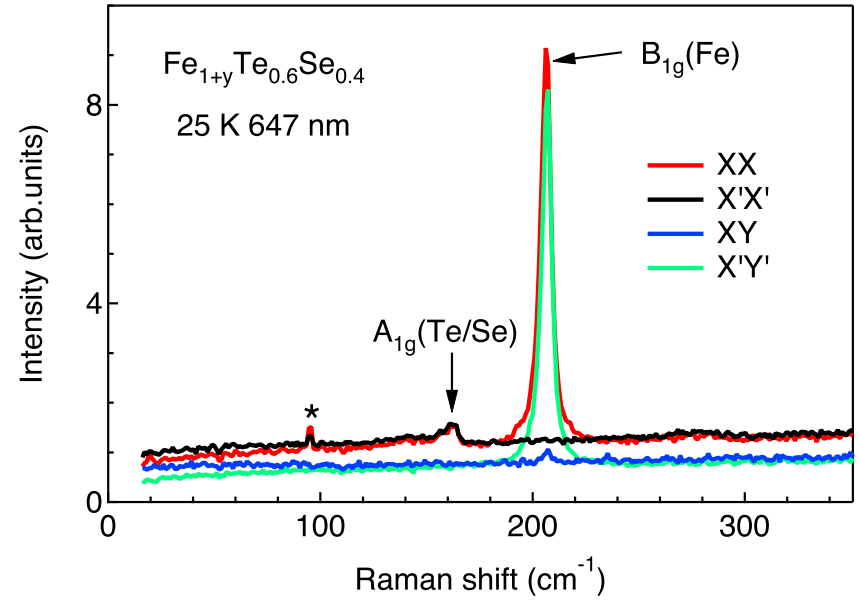

FIG. 2. Raman intensity of $\mathrm{Fe}_{1+y} \mathrm{Te}_{0.6} \mathrm{Se}_{0.4}$ at $25 \mathrm{~K}$ for $X X, X Y$, $X^{\prime} X^{\prime}$, and $X^{\prime} Y^{\prime}$ scattering geometries. The star marks a laser plasma line.

\section{B. Raman continuum}

Besides the phonon modes, in Fig. 3 we compare the Raman continuum above and below $T_{c}$ for three scattering geometries. It has been conjectured that the degeneracy of the partially filled $d_{x z} / d_{y z}$ orbitals is a necessary condition for anomalously strong nematic effects observed in FeSCs [16,30,34-42]: Such degeneracy enables dynamical charge oscillations at low frequencies that could give rise to a local fluctuating quadrupole moment with an amplitude proportional to the oscillating $d$-orbital occupation imbalance, $n_{x z}-$ $n_{y z}$. These soft quadrupole fluctuations often show critical behavior leading to a $d$-wave Pomeranchuk-like instability [16]. For the majority of FeSCs such fluctuations most dramatically manifest themselves in anomalously strong $X Y$-symmetry Raman response as an overdamped quasielastic feature in the normal state $[16,30,37,41]$ which undergoes a metamorphosis into a coherent in-gap collective mode below $T_{c}$ $[16,17,43]$. The same observation holds also for the $\mathrm{FeSe}_{1-x} \mathrm{~S}_{x}$ alloys [31].

It is interesting to note that the $\mathrm{Fe}_{1+y} \mathrm{Te}_{0.6} \mathrm{Se}_{0.4}$ alloy is one of the exceptions: while the elastoresistance probe unambiguously detects strong nematic fluctuations of the $B_{2 g}$ symmetry [34], the Raman response in $B_{2 g}$ channels remains quite weak (Fig. 3). A possible explanation for such an unexpected contradiction was proposed in a recent study [44] where the authors consider effects of constructive versus destructive interference of the Raman amplitudes originating from the

TABLE I. Comparison of the phonon frequencies $\left(\omega_{\mathrm{ph}}\right)$ and the linewidths $(\gamma)$ for $A_{1 g}(\mathrm{Te} / \mathrm{Se})$ and $B_{1 g}(\mathrm{Fe})$ modes at $5 \mathrm{~K}$ for $\mathrm{Fe}_{1+y} \mathrm{Te}_{1-x} \mathrm{Se}_{x}$ to the studies with similar compositions. Units are in $\mathrm{cm}^{-1}$.

\begin{tabular}{lcccc}
\hline \hline Sample & $\omega_{A_{1 g}}$ & $\gamma_{A_{1 g}}$ & $\omega_{B_{1 g}}$ & $\gamma_{B_{1 g}}$ \\
\hline $\mathrm{FeTe}_{0.6} \mathrm{Se}_{0.4}[24]$ & 162.6 & 27.2 & 207.7 & 8.8 \\
$\mathrm{Fe}_{0.95} \mathrm{Te}_{0.56} \mathrm{Se}_{0.44}[23]$ & 162 & 20 & 207 & 5 \\
$\mathrm{Fe}_{1+y} \mathrm{Te}_{0.6} \mathrm{Se}_{0.4}$ (this work) & 162 & 6.9 & 208.2 & 4 \\
\hline \hline
\end{tabular}




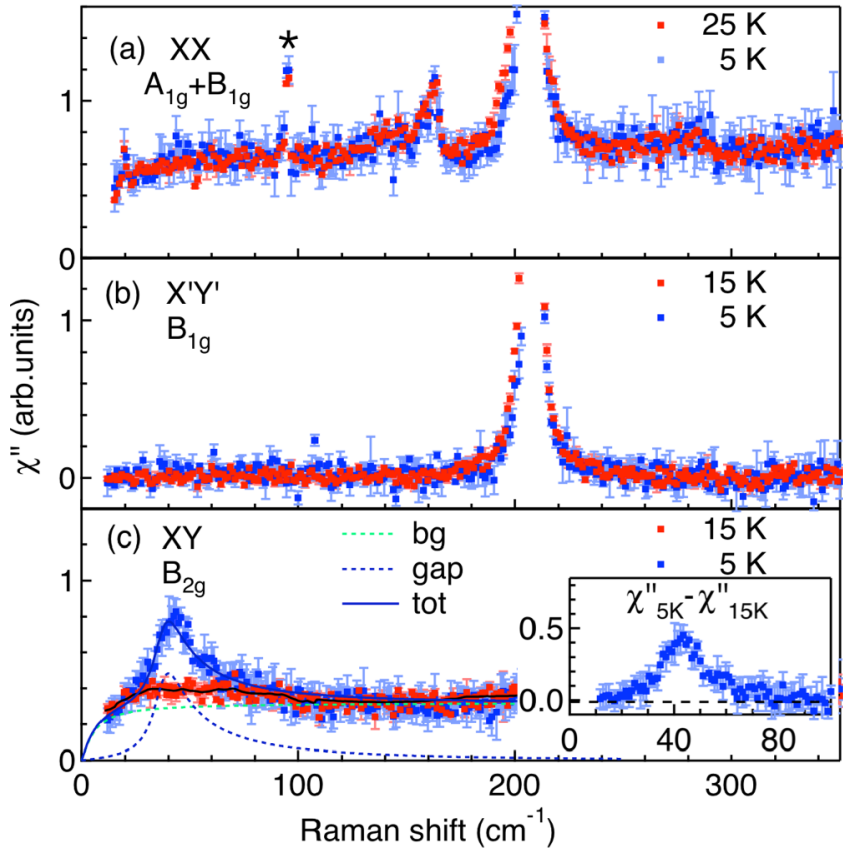

FIG. 3. Raman response of $\mathrm{Fe}_{1+y} \mathrm{Te}_{0.6} \mathrm{Se}_{0.4}$ above and below $T_{c}$ in $X X$ (a), $X^{\prime} Y^{\prime}$ (b), and $X Y$ (c) scattering geometries. The error bars represent one standard deviation. The star in (a) marks a laser plasma line. The solid black line in (c) represents a smoothed curve for the Raman response at $15 \mathrm{~K}$. The difference between the response above and below $T_{c}$ is shown in the inset. The blue and green dashed lines represent the components of the isotropic gap and a background resulting from a fit of Raman response at $5 \mathrm{~K}$ to an isotropic gap function $[20,45,46]$.

scattering at two FS pockets in the vicinity of the $\Gamma$ and the $M$ points: the interference signal is constructive if the FS distortions due to nematic Pomeranchuk-like fluctuations at $\Gamma$ and $M$ pockets are in antiphase, and it is destructive if it is in-phase. Hence, the sign of the interaction between these two pockets for $\mathrm{Fe}_{1+y} \mathrm{Te}_{0.6} \mathrm{Se}_{0.4}$ could be distinct from the majority of FeSCs compounds for which both the $B_{2 g}$ symmetry elastoresistance and the $B_{2 g}$ Raman response demonstrate the same singular behavior.

\section{The superconducting state}

Before looking into the Raman features observed in the SC state, we recall the SC gap values obtained by complementary spectroscopic probes. The ARPES revealed nodeless SC gaps with small or negligible in-plane anisotropy on both holelike and electronlike FS pockets $[27,28,47]$. In addition to the SC gap of $1.8 \mathrm{meV}$ for the topological surface state [28], two close to isotropic gaps for bulk bands have been reported: a $3 \mathrm{meV}$ gap for the holelike FS pocket with $d_{x z} / d_{y z}$ character centered around the $\Gamma$ point [27] and a larger, about $4.2 \mathrm{meV}$ gap for the electron pocket around the $M$ point [47]. A scanning tunneling spectroscopy (STS) study has reported a $\mathrm{SC}$ coherence peak at $1.5 \mathrm{meV}$ and a shoulder at $2.5 \mathrm{meV}$ [48]. An infrared spectroscopy (IR) study has derived from the scattering rate analysis two SC gap features at 2.47 and $5.08 \mathrm{meV}$ [49]. Measurements of the specific heat have confirmed the nodeless nature of the SC order parameter with an estimate
TABLE II. The summary of the values for the SC gaps and the bosonic mode deducted from ARPES, STS, IR, specific heat, Raman scattering, and INS measurements for $\mathrm{Fe}_{1+y} \mathrm{Te}_{0.6} \mathrm{Se}_{0.4}$ with similar compositions. $\Delta_{1}, \Delta_{2}$, and $\Delta_{3}$ are gap values for the surface state, the hole FS, and electron FS pockets, respectively. $E_{C M}$ is the energy of the bosonic collective mode. All energies are given in units of $\mathrm{meV}$.

\begin{tabular}{lcccc}
\hline \hline & $\Delta_{1}$ (surface) & $\Delta_{2}$ (hole) & $\Delta_{3}($ elec $)$ & $E_{C M}$ \\
\hline ARPES & $1.8[28]$ & $3[27]$ & $4.2[47]$ & \\
STS & $1.5[48,52]$ & $2.5[48]$ & & \\
IR & & $2.47[49]$ & $5.08[49]$ & \\
Specific heat & & $2.3[50]$ & & \\
Raman & & 2.8 (this work) & \\
INS & & & $6.5[51]$ \\
\hline \hline
\end{tabular}

of the averaged gap value at $2.3 \mathrm{meV}$ [50]. An inelastic neutron scattering (INS) study has reported a spin-resonance mode at $\mathbf{Q}=(\pi, \pi)$ and $6.5 \mathrm{meV}$, the energy consistent with the sum of the gap values on the hole and electron pockets [51]. The values of the gaps and the bosonic mode deduced from different spectroscopies are summarized in Table II.

In Fig. 3 we compare the Raman response above and below $T_{c}$ for three scattering geometries. For the $X X$ and $X^{\prime} \mathrm{Y}^{\prime}$ scattering geometries, the electronic continuum barely changes upon cooling below $T_{c}$. In contrast, for $X Y$ polarization a clear peak at around $45 \mathrm{~cm}^{-1}$ emerges below $T_{c}$. We relate this peak to the pair-breaking excitation. Since the peak's energy is at about $5.6 \mathrm{meV}$, based on Table II we assign it to the gap $2 \Delta_{2}$ on the holelike FS pocket derived from the iron $d_{x z} / d_{y z}$ orbitals [29] with a typical gap value $\Delta_{2} \approx 3 \mathrm{meV}$ from ARPES measurement preformed on samples from the same batch [27].

In Fig. 3(c) we show a fit to the Raman response data in $X Y$ polarization at $5 \mathrm{~K}$ with a single BCS coherence peak function $\chi^{\prime \prime} \sim 4 \Delta^{2} /\left(\omega \sqrt{\omega^{2}-4 \Delta^{2}}\right)$ resulting from the fundamental bulk gap at the value $2 \Delta=38 \mathrm{~cm}^{-1}$ broadened by $7 \mathrm{~cm}^{-1}$ $[20,45,46]$.

We do not detect pair-breaking excitation for the electron pocket at larger than the fundamental gap energy. The reason for that could be again the destructive interference between the Raman field-driven amplitudes due to scattering from the two FS pockets if the fluctuations at $\Gamma$ and $M$ pockets are inphase [44]. In addition, the pair-breaking excitation across a larger than the fundamental gap is expected to be broadened due to much faster quasiparticle relaxation.

\section{Phonon self-energy effects}

The frequency and linewidth temperature dependence for $B_{1 g}(\mathrm{Fe})$ phonon mode are presented in Figs. 4(b) and 4(c). Above $T_{c}$, the mode displays behavior consistent with a standard anharmonic decay model [53-55]. Below $T_{c}$, in contrast to the $A_{1 g}(\mathrm{Te} / \mathrm{Se})$ phonon which changes little upon cooling across $T_{c}$ (the frequency changes less than one $\mathrm{cm}^{-1}$ while the linewidth barely changes) [inset of Fig. 4(a)], the $B_{1 g}(\mathrm{Fe})$ mode shows abnormal behavior on the cooling into 

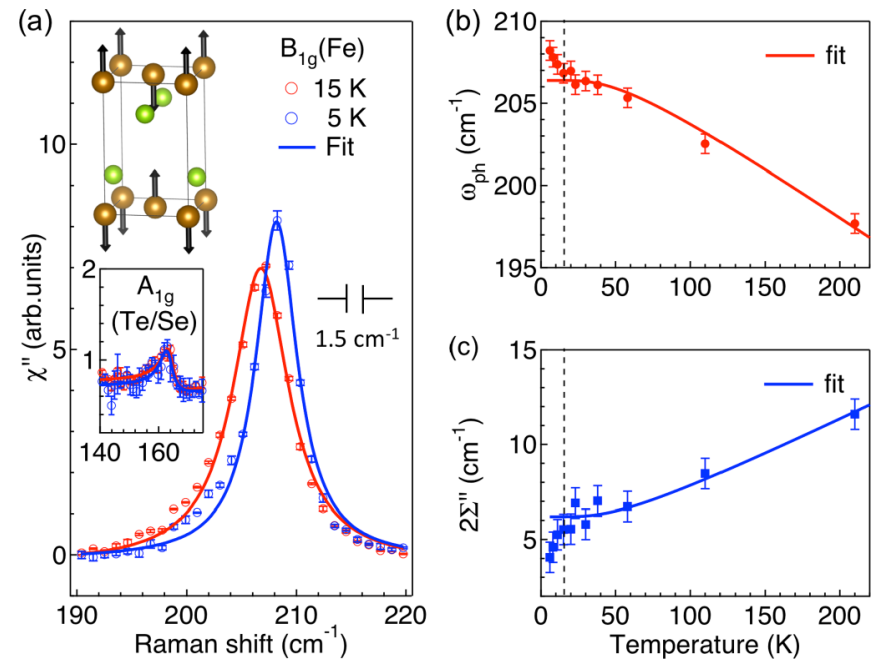

FIG. 4. (a) Raman response in $X^{\prime} Y^{\prime}$ polarization for $B_{1 g}(\mathrm{Fe})$ phonon mode at 15 (red) and $5 \mathrm{~K}$ (blue). The top inset illustrates the mode's atomic displacement. The bottom inset displays the $A_{1 g}(\mathrm{Te} / \mathrm{Se})$ phonon mode at $25 \mathrm{~K}$ and $5 \mathrm{~K}$ measured in $X X$ polarization. The solid lines are fits to the phonon data. (b) and (c) $\mathrm{T}$ dependence of the $B_{1 g}(\mathrm{Fe})$ phonon frequency $\omega_{\mathrm{ph}}$ and linewidth $2 \Sigma^{\prime \prime}$. The solid lines represent the fitting of the phononic self-energy $\mathrm{T}$ dependence in the normal state by anharmonic decay model [55]. The dashed vertical lines represent $T_{c}$.

SC state: the mode's frequency and linewidth show additional hardening and sharpening [Fig. 4(a)].

The $B_{1 g}(\mathrm{Fe})$ phonon energy is about $26 \mathrm{meV}$, much larger than the twice of the maximum gap $2 \Delta_{3}=8.4 \mathrm{meV}$ [47]. Such hardening for a phonon with $\omega_{\mathrm{ph}}>2 \Delta$ was previously reported for $\mathrm{MgB}_{2}$ [56], for cuprate superconductors [57-62], and for FeSCs [18-20]. The effects were explained within the Zeyher-Zwicknagl's (ZZ) model [63] as a consequence of electron-phonon coupling. When the SC gap opens below $T_{c}$, the electronic density of states around the Fermi level is reorganized and pushed above the gap energy to the proximity of the phonon frequency resulting in phononic self-energy effects: the mode's broadening due to faster decay and shift to higher frequencies.

It is interesting to note here that contrary to the standard $\mathrm{ZZ}$ model expectation, the phonon linewidth for $\mathrm{Fe}_{1+y} \mathrm{Te}_{0.6} \mathrm{Se}_{0.4}$ further decreases in the SC state. A similar $B_{1 g}(\mathrm{Fe})$ mode sharpening at the entrance in the SC state was noted for $\mathrm{NaFe}_{0.97} \mathrm{Co}_{0.03} \mathrm{As}$ [19], $\mathrm{Ba}_{0.72} \mathrm{~K}_{0.28} \mathrm{Fe}_{2} \mathrm{As}_{2}$ [18], and $\mathrm{Sr}_{0.85} \mathrm{~K}_{0.15} \mathrm{Fe}_{2} \mathrm{As}_{2}$ [18]. A possible reason for such reduction of the phonon scattering rate could be due to the discussed above destructive interference in the Raman signal of a multiband system which results in reduction of the electronic Raman spectral weight at the phonon mode frequency $\omega_{\mathrm{ph}}$ even when the mode is above the SC gap value.

To quantitatively estimate the SC induced self-energy effects and to compute the electron-phonon coupling constant, we fit the temperature dependent parameters of $B_{1 g}(\mathrm{Fe})$ phonon: $\chi_{\mathrm{ph}}^{\prime \prime}(\omega) \propto 4 \omega_{0}^{2} \Sigma^{\prime \prime}\left[\left(\omega^{2}-\omega_{0}^{2}-2 \omega_{0} \Sigma^{\prime}\right)^{2}+\right.$ $\left.4\left(\omega_{0} \Sigma^{\prime \prime}\right)^{2}\right]^{-1}$, where $\omega_{0}$ is the bare phonon frequency that is derived from an extrapolation of the frequency fit in normal state to anharmonic decay model [55]; here $\Sigma=\Sigma^{\prime}+i \Sigma^{\prime \prime}$ is complex phonon self-energy [63]. The phonon appears at $\omega_{\mathrm{ph}}=\sqrt{\omega_{0}^{2}+2 \omega_{0} \Sigma^{\prime}}$ if $\Sigma^{\prime \prime}$ is small. The fitting results below $T_{c}$ are presented in Figs. 4(b) and 4(c).

We apply the $\mathrm{ZZ}$ model to compute the electron-phonon coupling constant [64]: $\quad \lambda \Gamma_{B_{1 g}}=-\kappa \sin u / u$, where $\kappa=\left(\left[\Sigma^{\prime}(5 K)-\Sigma^{\prime}(15 K)\right]-i\left[\Sigma^{\prime \prime}(5 K)-\Sigma^{\prime \prime}(15 K)\right]\right) / \omega_{\mathrm{ph}}$ $(15 K)$ and $u \equiv \pi+2 i \cosh ^{-1}\left[\omega_{\mathrm{ph}}(15 K) / 2 \Delta\right]$. Using $2 \Delta=45 \mathrm{~cm}^{-1}$ obtained from the pair-breaking peak energy in the $B_{2 g}$ channel, we derive a weak electron-phonon coupling constant $\lambda_{B_{1 g}}^{\Gamma} \approx 0.026$. Since the $A_{1 g}(\mathrm{Te} / \mathrm{Se})$ phonon mode shows only a minor renormalization [inset of Fig. 4(a)], the $B_{1 g}(\mathrm{Fe})$ phonon is the only mode that shows a noticeable self-energy effect. Therefore, we use $\lambda_{B_{1 g}}$ as an estimate for the averaged electron-phonon coupling constant $\lambda^{\Gamma}$ at the BZ zone center [20].

By comparison, a much larger electron-phonon coupling constant $\lambda^{\Gamma} \approx 0.3$ was reported for a conventional phononmediated superconductor $\mathrm{MgB}_{2}$ with $T_{c}=39 \mathrm{~K}$ [56]. Furthermore, for $\mathrm{V}_{3} \mathrm{Ga}$, an $s$-wave superconductor with a similar $T_{c}=14.2 \mathrm{~K}$, the electron-phonon coupling constant was estimated to be $\lambda \approx 0.9$ by optical measurements [65]. Therefore, the electron-phonon coupling constant $\lambda^{\Gamma} \approx 0.026$ is clearly insufficient to cause $T_{c}=14 \mathrm{~K}$ superconductivity in $\mathrm{Fe}_{1+y} \mathrm{Te}_{0.6} \mathrm{Se}_{0.4}$.

\section{CONCLUSIONS}

In summary, we present a polarization-resolved Raman study of $\mathrm{Fe}_{1+y} \mathrm{Te}_{0.6} \mathrm{Se}_{0.4}$ superconductor with $T_{c}=14 \mathrm{~K}$. In the SC state we detect a distinct pair-breaking excitation across the fundamental gap at $45 \mathrm{~cm}^{-1}(2 \Delta=5.6 \mathrm{meV})$ in the $B_{2 g}$ symmetry channel. This energy is consistent with the gap that was reported by ARPES studies for the holelike FS pocket around the $\Gamma$ point that is derived from $d_{x z} / d_{y z}$ iron orbitals.

We analyze the superconductivity-induced phonon selfenergy effects for the $B_{1 g}(\mathrm{Fe})$ mode and compute the electronphonon coupling constant $\lambda^{\Gamma} \approx 0.026$, which is too small to explain superconductivity with $T_{c}=14 \mathrm{~K}$. We also note an anomalous phonon mode sharpening at cooling into superconducting state and propose a multiband interference effect that may cause such anomalous reduction in the scattering rate.

\section{ACKNOWLEDGMENTS}

We thank A. Chubukov, L. Y. Kong, and J. X. Yin for useful discussions. The spectroscopic work at Rutgers (S.W., A.L., and G.B.) was supported by NSF Grant No. DMR-1709161. Crystal growth and characterization at Technion-Israel Institute of Technology (A.A., I.F., and A.K.) were supported by Israel Science Foundation Grant No. 320/17. The work at NICPB was supported by Estonian Research Council Grant No. PRG736. 
[1] Y. Kamihara, T. Watanabe, M. Hirano, and H. Hosono, Iron-based layered superconductor $\mathrm{LaO}_{1-x} \mathrm{~F}_{x} \mathrm{FeAs}(x=0.05-$ 0.12) with $T_{c}=26 \mathrm{~K}$, J. Am. Chem. Soc. 130, 3296 (2008).

[2] J. Paglione and R. L. Greene, High-temperature superconductivity in iron-based materials, Nat. Phys. 6, 645 (2010).

[3] F. Wang and D.-H. Lee, The electron-pairing mechanism of iron-based superconductors, Science 332, 200 (2011).

[4] R. M. Fernandes and A. V. Chubukov, Low-energy microscopic models for iron-based superconductors: A review, Rep. Prog. Phys. 80, 014503 (2016).

[5] P. J. Hirschfeld, Using gap symmetry and structure to reveal the pairing mechanism in Fe-based superconductors, C. R. Phys. 17, 197 (2016).

[6] P. C. Dai, Antiferromagnetic order and spin dynamics in ironbased superconductors, Rev. Mod. Phys. 87, 855 (2015).

[7] Q. M. Si, R. Yu, and E. Abrahams, High-temperature superconductivity in iron pnictides and chalcogenides, Nat. Rev. Mater. 1, 16017 (2016).

[8] P. Coleman, Y. Komijani, and E. Knig, The triplet resonating valence bond state and superconductivity in Hund's metals, arXiv: 1910.03168.

[9] P. Richard, T. Qian, and H. Ding, ARPES measurements of the superconducting gap of Fe-based superconductors and their implications to the pairing mechanism, J. Phys.: Condens. Matter 27, 293203 (2015).

[10] M. H. Fang, H. M. Pham, B. Qian, T. J. Liu, E. K. Vehstedt, Y. Liu, L. Spinu, and Z. Q. Mao, Superconductivity close to magnetic instability in $\mathrm{Fe}\left(\mathrm{Se}_{1-x} \mathrm{Te}_{x}\right)_{0.82}$, Phys. Rev. B 78, 224503 (2008).

[11] B. Muschler, W. Prestel, R. Hackl, T. P. Devereaux, J. G. Analytis, J.-H. Chu, and I. R. Fisher, Band- and momentum-dependent electron dynamics in superconducting $\mathrm{Ba}\left(\mathrm{Fe}_{1-x} \mathrm{Co}_{x}\right)_{2} \mathrm{As}_{2}$ as seen via electronic Raman scattering, Phys. Rev. B 80, 180510(R) (2009).

[12] L. Chauvière, Y. Gallais, M. Cazayous, M. A. Méasson, A. Sacuto, D. Colson, and A. Forget, Impact of the spin-densitywave order on the superconducting gap of $\mathrm{Ba}\left(\mathrm{Fe}_{1-x} \mathrm{Co}_{x}\right)_{2} \mathrm{As}_{2}$, Phys. Rev. B 82, 180521(R) (2010).

[13] P. Massat, D. Farina, I. Paul, S. Karlsson, P. Strobel, P. Toulemonde, M.-A. Méasson, M. Cazayous, A. Sacuto, S. Kasahara, Y. Shibauchi, T. Matsuda, and Y. Gallais, Chargeinduced nematicity in FeSe, Proc. Natl. Acad. Sci. USA 113, 9177 (2016).

[14] S. Sugai, Y. Mizuno, K. Kiho, M. Nakajima, C. H. Lee, A. Iyo, H. Eisaki, and S. Uchida, Pairing symmetry of the multiorbital pnictide superconductor $\mathrm{BaFe}_{1.84} \mathrm{Co}_{0.16} \mathrm{As}_{2}$ from Raman scattering, Phys. Rev. B 82, 140504(R) (2010).

[15] T. Böhm, A. F. Kemper, B. Moritz, F. Kretzschmar, B. Muschler, H.-M. Eiter, R. Hackl, T. P. Devereaux, D. J. Scalapino, and H.-H. Wen, Balancing Act: Evidence for a Strong Subdominant $d$-Wave Pairing Channel in $\mathrm{Ba}_{0.6} \mathrm{~K}_{0.4} \mathrm{Fe}_{2} \mathrm{As}_{2}$, Phys. Rev. X 4, 041046 (2014).

[16] V. K. Thorsmølle, M. Khodas, Z. P. Yin, C. Zhang, S. V. Carr, P. Dai, and G. Blumberg, Critical quadrupole fluctuations and collective modes in iron pnictide superconductors, Phys. Rev. B 93, 054515 (2016).

[17] S.-F. Wu, P. Richard, H. Ding, H.-H. Wen, G. Tan, M. Wang, C. Zhang, P. Dai, and G. Blumberg, Superconductivity and electronic fluctuations in $\mathrm{Ba}_{1-x} \mathrm{~K}_{x} \mathrm{Fe}_{2} \mathrm{As}_{2}$ studied by Raman scattering, Phys. Rev. B 95, 085125 (2017).

[18] K.-Y. Choi, P. Lemmens, I. Eremin, G Zwicknagl, H. Berger, G. L. Sun, D. L. Sun, and C. T. Lin, Self-energy effects and electron-phonon coupling in FeAs superconductors, J. Phys.: Condens. Matter 22, 115802 (2010).

[19] Y. J. Um, Y. Bang, B. H. Min, Y. S. Kwon, and M. Le Tacon, Superconductivity-induced phonon renormalization on $\mathrm{NaFe}_{1-x} \mathrm{Co}_{x} \mathrm{As}$, Phys. Rev. B 89, 184510 (2014).

[20] W.-L. Zhang, W. R. Meier, T. Kong, P. C. Canfield, and G. Blumberg, High- $T_{c}$ superconductivity in $\mathrm{CaKFe}_{4} \mathrm{As}_{4}$ in absence of nematic fluctuations, Phys. Rev. B 98, 140501(R) (2018).

[21] T.-L. Xia, D. Hou, S. C. Zhao, A. M. Zhang, G. F. Chen, J. L. Luo, N. L. Wang, J. H. Wei, Z.-Y. Lu, and Q. M. Zhang, Raman phonons of $\alpha$-FeTe and $\mathrm{Fe}_{1.03} \mathrm{Se}_{0.3} \mathrm{Te}_{0.7}$ single crystals, Phys. Rev. B 79, 140510(R) (2009).

[22] V. Gnezdilov, Y. Pashkevich, P. Lemmens, A. Gusev, K. Lamonova, T. Shevtsova, I. Vitebskiy, O. Afanasiev, S. Gnatchenko, V. Tsurkan, J. Deisenhofer, and A. Loidl, Anomalous optical phonons in FeTe chalcogenides: Spin state, magnetic order and lattice anharmonicity, Phys. Rev. B 83, 245127 (2011).

[23] Y. J. Um, A. Subedi, P. Toulemonde, A. Y. Ganin, L. Boeri, M. Rahlenbeck, Y. Liu, C. T. Lin, S. J. E. Carlsson, A. Sulpice, M. J. Rosseinsky, B. Keimer, and M. Le Tacon, Anomalous dependence of $c$-axis polarized $\mathrm{Fe} \mathrm{B}_{1 g}$ phonon mode with $\mathrm{Fe}$ and Se concentrations in $\mathrm{Fe}_{1+y} \mathrm{Te}_{1-x} \mathrm{Se}_{x}$, Phys. Rev. B 85, 064519 (2012).

[24] K. Okazaki, S. Sugai, S. Niitaka, and H. Takagi, Phonon, twomagnon, and electronic Raman scattering of $\mathrm{Fe}_{1+y} \mathrm{Te}_{1-x} \mathrm{Se}_{x}$, Phys. Rev. B 83, 035103 (2011).

[25] Z. V. Popovi, N. Lazarevi, S. Bogdanovi, M. M. Radonji, D. Tanaskovi, R. Hu, H. Lei, and C. Petrovic, Signatures of the spin-phonon coupling in $\mathrm{Fe}_{1+y} \mathrm{Te}_{1 x} \mathrm{Se}_{x}$ alloys, Solid State Commun. 193, 51 (2014).

[26] The lack of pair-breaking excitation detection in the earlier studies is likely due to sample contamination signatures of which include a significant low-frequency tail in the data [22-24], broad $A_{1 g}$ phonons [22,24], and the $A_{1 g}$ modes' intensity "leakage" into forbidden polarizations $[22,24]$.

[27] S. Rinott, K. B. Chashka, A. Ribak, Emile D. L. Rienks, A. Taleb-Ibrahimi, P. Le Fevre, F. Bertran, M. Randeria, and A. Kanigel, Tuning across the BCS-BEC crossover in the multiband superconductor $\mathrm{Fe}_{1+y} \mathrm{Se}_{x} \mathrm{Te}_{1-x}$ : An angle-resolved photoemission study, Sci. Adv. 3, e1602372 (2017).

[28] P. Zhang, K. Yaji, T. Hashimoto, Y. Ota, T. Kondo, K. Okazaki, Z. J. Wang, J. S. Wen, G. D. Gu, H. Ding, and S. Shin, Observation of topological superconductivity on the surface of an iron-based superconductor, Science 360, 182 (2018).

[29] P. Zhang, Z. J. Wang, X. X. Wu, K. Yaji, Y. Ishida, Y. Kohama, G. Y. Dai, Y. Sun, C. Bareille, K. Kuroda, T. Kondo, K. Okazaki, K. Kindo, X. C. Wang, C. Q. Jin, J. P. Hu, R. Thomale, K. Sumida, S. L. Wu, K. Miyamoto, T. Okuda, H. Ding, G. D. Gu, T. Tamegai, T. Kawakami, M. Sato, and S. Shin, Multiple topological states in iron-based superconductors, Nat. Phys. 15, 41 (2019).

[30] F. Kretzschmar, T. Bohm, U. Karahasanovic, B. Muschler, A. Baum, D. Jost, J. Schmalian, S. Caprara, M. Grilli, C. Di Castro, J. G. Analytis, J.-H. Chu, I. R. Fisher, and 
R. Hackl, Critical spin fluctuations and the origin of nematic order in $\mathrm{Ba}\left(\mathrm{Fe}_{1-x} \mathrm{Co}_{x}\right)_{2} \mathrm{As}_{2}$, Nat. Phys. 12, 560 (2016).

[31] W.-L. Zhang, S.-F. Wu, S. Kasahara, T. Shibauchi, Y. Matsuda, and G. Blumberg, Stripe quadrupole order in the nematic phase of $\mathrm{FeSe}_{1-x} \mathrm{~S}_{x}$, arXiv:1710.09892.

[32] T. P. Devereaux and R. Hackl, Inelastic light scattering from correlated electrons, Rev. Mod. Phys. 79, 175 (2007).

[33] The phonon frequency for both modes and the linewidth for the $B_{1 g}(\mathrm{Fe})$ mode are consistent with Refs. [23,24]. In contrast, the linewidth for the $A_{1 g}(\mathrm{Te} / \mathrm{Se})$ mode in this study is significantly sharper than in Refs. [23,24].

[34] H. H. Kuo, J. H. Chu, J. C. Palmstrom, S. A. Kivelson, and I. R. Fisher, Ubiquitous signatures of nematic quantum criticality in optimally doped Fe-based superconductors, Science 352, 958 (2016).

[35] X. Y. Lu, J. T. Park, R. Zhang, H. Q. Luo, A. H. Nevidomskyy, Q. M. Si, and P. C. Dai, Nematic spin correlations in the tetragonal state of uniaxial-strained $\mathrm{BaFe}_{2-x} \mathrm{Ni}_{x} \mathrm{As}_{2}$, Science 345, 657 (2014).

[36] A. E. Böhmer and C. Meingast, Electronic nematic susceptibility of iron-based superconductors, C. R. Phys. 17, 90 (2016).

[37] Y. Gallais, R. M. Fernandes, I. Paul, L. Chauvière, Y.-X. Yang, M.-A. Méasson, M. Cazayous, A. Sacuto, D. Colson, and A. Forget, Observation of Incipient Charge Nematicity in $\mathrm{Ba}\left(\mathrm{Fe}_{1-x} \mathrm{Co}_{x}\right)_{2} \mathrm{As}_{2}$, Phys. Rev. Lett. 111, 267001 (2013).

[38] Y. Gallais and I. Paul, Charge nematicity and electronic Raman scattering in iron-based superconductors, C. R. Phys. 17, 113 (2016).

[39] S. H. Baek, D. V. Efremov, J. M. Ok, J. S. Kim, J. van den Brink, and B. Büchner, Orbital-driven nematicity in FeSe, Nat. Mater. 14, 210 (2015).

[40] A. P. Dioguardi, T. Kissikov, C. H. Lin, K. R. Shirer, M. M. Lawson, H.-J. Grafe, J.-H. Chu, I. R. Fisher, R. M. Fernandes, and N. J. Curro, NMR Evidence for Inhomogeneous Nematic Fluctuations in $\mathrm{BaFe}_{2}\left(\mathrm{As}_{1-x} \mathrm{P}_{x}\right)_{2}$, Phys. Rev. Lett. 116, 107202 (2016).

[41] N. Lazarevic and R. Hackl, Fluctuations and pairing in Fe-based superconductors: Light scattering experiments, arXiv:1909.00173.

[42] R. M. Fernandes, A. V. Chubukov, and J. Schmalian, What drives nematic order in iron-based superconductors? Nat. Phys. 10, 97 (2014).

[43] Y. Gallais, I. Paul, L. Chauvière, and J. Schmalian, Nematic Resonance in the Raman Response of Iron-Based Superconductors, Phys. Rev. Lett. 116, 017001 (2016).

[44] M. Udina, M. Grilli, L. Benfatto, and A. Chubukov, Raman response in the nematic phase of FeSe, arXiv:1908.11361.

[45] M. V. Klein and S. B. Dierker, Theory of Raman scattering in superconductors, Phys. Rev. B 29, 4976 (1984).

[46] G. Blumberg, A. Mialitsin, B. S. Dennis, N. D. Zhigadlo, and J. Karpinski, Multi-gap superconductivity in $\mathrm{MgB}_{2}$ : MagnetoRaman spectroscopy, Physica C 456, 75 (2007).

[47] H. Miao, P. Richard, Y. Tanaka, K. Nakayama, T. Qian, K. Umezawa, T. Sato, Y.-M. Xu, Y. B. Shi, N. Xu, X.-P. Wang, P. Zhang, H.-B. Yang, Z.-J. Xu, J. S. Wen, G.-D. Gu, X. Dai, J.-P. Hu, T. Takahashi, and H. Ding, Isotropic superconducting gaps with enhanced pairing on electron Fermi surfaces in $\mathrm{FeTe}_{0.55} \mathrm{Se}_{0.45}$, Phys. Rev. B 85, 094506 (2012).
[48] J.-X. Yin, Z. Wu, J.-H. Wang, Z.-Y. Ye, J. Gong, X.-Y. Hou, L. Shan, A. Li, X.-J. Liang, X.-X. Wu, J. Li, C.-S. Ting, Z.-Q. Wang, J.-P. Hu, P.-H. Hor, H. Ding, and S. H. Pan, Observation of a robust zero-energy bound state in iron-based superconductor Fe(Te,Se), Nat. Phys. 11, 543 (2015).

[49] C. C. Homes, A. Akrap, J. S. Wen, Z. J. Xu, Z. W. Lin, Q. Li, and G. D. Gu, Electronic correlations and unusual superconducting response in the optical properties of the iron chalcogenide $\mathrm{FeTe}_{0.55} \mathrm{Se}_{0.45}$, Phys. Rev. B 81, 180508(R) (2010).

[50] R. Escudero and R. E. López-Romero, The energy gap of the compound $\mathrm{FeSe}_{0.5} \mathrm{Te}_{0.5}$ determined by specific heat and point contact spectroscopy, Solid State Commun. 220, 21 (2015).

[51] Y. M. Qiu, W. Bao, Y. Zhao, C. Broholm, V. Stanev, Z. Tesanovic, Y. C. Gasparovic, S. Chang, J. Hu, B. Qian, M. H. Fang, and Z. Q. Mao, Spin Gap and Resonance at the Nesting Wave Vector in Superconducting $\mathrm{FeSe}_{0.4} \mathrm{Te}_{0.6}$, Phys. Rev. Lett. 103, 067008 (2009).

[52] T. Hanaguri, S. Niitaka, K. Kuroki, and H. Takagi, Unconventional s-wave superconductivity in $\mathrm{Fe}(\mathrm{Se}, \mathrm{Te})$, Science 328, 474 (2010).

[53] P. G. Klemens, Anharmonic decay of optical phonons, Phys. Rev. 148, 845 (1966).

[54] J. Menéndez and M. Cardona, Temperature dependence of the first-order Raman scattering by phonons in $\mathrm{Si}, \mathrm{Ge}$, and $\alpha-\mathrm{Sn}$ : Anharmonic effects, Phys. Rev. B 29, 2051 (1984).

[55] The temperature dependence of frequency and linewidth is fitted by $\omega_{\mathrm{ph}}(T)=\omega_{0}-C\left[1+2 /\left(e^{\hbar \omega_{0} / 2 k_{B} T}-1\right)\right]$ and $\Gamma_{\mathrm{ph}}(T)=$ $\Gamma_{0}+\Gamma_{1}\left[1+2 /\left(e^{\hbar \omega_{0} / 2 k_{B} T}-1\right)\right]$. The fit results in parameters $\omega_{0}=211.1 \mathrm{~cm}^{-1}, C=4.8 \mathrm{~cm}^{-1}, \Gamma_{0}=3.2 \mathrm{~cm}^{-1}$, and $\Gamma_{1}=2.9 \mathrm{~cm}^{-1}$.

[56] A. Mialitsin, B. S. Dennis, N. D. Zhigadlo, J. Karpinski, and G. Blumberg, Anharmonicity and self-energy effects of the $E_{2 g}$ phonon in $\mathrm{MgB}_{2}$, Phys. Rev. B 75, 020509(R) (2007).

[57] S. L. Cooper, M. V. Klein, B. G. Pazol, J. P. Rice, and D. M. Ginsberg, Raman scattering from superconducting gap excitations in single-crystal $\mathrm{YBa}_{2} \mathrm{Cu}_{3} \mathrm{O}_{7-\delta}$, Phys. Rev. B 37, 5920 (1988).

[58] C. Thomsen, M. Cardona, B. Gegenheimer, R. Liu, and A. Simon, Untwinned single crystals of $\mathrm{YBa}_{2} \mathrm{Cu}_{3} \mathrm{O}_{7-\delta}$ : An optical investigation of the $a-b$ anisotropy, Phys. Rev. B 37, 9860 (1988).

[59] B. Friedl, C. Thomsen, and M. Cardona, Determination of the Superconducting Gap in $\mathrm{RBa}_{2} \mathrm{Cu}_{3} \mathrm{O}_{7-\delta}$, Phys. Rev. Lett. 65, 915 (1990).

[60] K. F. McCarty, H. B. Radousky, J. Z. Liu, and R. N. Shelton, Temperature dependence of the linewidths of the Raman-active phonons of $\mathrm{YBa}_{2} \mathrm{Cu}_{3} \mathrm{O}_{7}$ : Evidence for a superconducting gap between 440 and $500 \mathrm{~cm}^{-1}$, Phys. Rev. B 43, 13751 (1991).

[61] G. Blumberg, M. V. Klein, L. Börjesson, R. Liang, and W. N. Hardy, Investigation of the temperature dependence of electron and phonon Raman scattering in single crystal $\mathrm{YBa}_{2} \mathrm{Cu}_{3} \mathrm{O}_{6.952}$, J. Supercond. 7, 445 (1994).

[62] C. Thomsen and M. Cardona, Raman scattering in high- $T_{c}$ superconductors, in Physical Properties of High Temperature Superconductors I, edited by D. M. Ginsberg (World Scientific, Singapore, 1998), pp. 409-507. 
[63] R. Zeyher and G. Zwicknagl, Superconductivity-induced phonon self-energy effects in high- $T_{c}$ superconductors, Z. Phys. B: Condens. Matter 78, 175 (1990).

[64] C. O. Rodriguez, A. I. Liechtenstein, I. I. Mazin, O. Jepsen, O. K. Andersen, and M. Methfessel, Optical near-zone-center phonons and their interaction with electrons in $\mathrm{YBa}_{2} \mathrm{Cu}_{3} \mathrm{O}_{7}$ :
Results of the local-density approximation, Phys. Rev. B 42, 2692 (1990).

[65] E. G. Maksimov and G. P. Motulevice, Determination of the electron-phonon coupling constant from optical measurements, Zh. Eksp. Teor. Fiz. 61, 414 (1972) [Sov. Phys. JETP 34, 219 (1972)]. 